\title{
Uncertainty Analysis and Model Reduction Based Global Optimisation of Distributed Large-scale Systems
}

\section{Document Version}

Accepted author manuscript

Link to publication record in Manchester Research Explorer

\section{Citation for published version (APA):}

Tao, M., \& Theodoropoulos, C. (2020). Uncertainty Analysis and Model Reduction Based Global Optimisation of Distributed Large-scale Systems. In 30th European Symposium on Computer Aided Chemical Engineering (1 ed., Vol. 47). Elsevier BV.

\section{Published in:}

30th European Symposium on Computer Aided Chemical Engineering

\section{Citing this paper}

Please note that where the full-text provided on Manchester Research Explorer is the Author Accepted Manuscript or Proof version this may differ from the final Published version. If citing, it is advised that you check and use the publisher's definitive version.

\section{General rights}

Copyright and moral rights for the publications made accessible in the Research Explorer are retained by the authors and/or other copyright owners and it is a condition of accessing publications that users recognise and abide by the legal requirements associated with these rights.

\section{Takedown policy}

If you believe that this document breaches copyright please refer to the University of Manchester's Takedown Procedures [http://man.ac.uk/04Y6Bo] or contact uml.scholarlycommunications@manchester.ac.uk providing relevant details, so we can investigate your claim.

\section{OPEN ACCESS}


Sauro Pierucci, Flavio Manenti, Giulia Bozzano, Davide Manca (Eds.)

PROCEEDINGS OF THE $30^{\text {th }}$ European Symposium on Computer Aided Process Engineering

(ESCAPE30), May 24-27, 2020, Milano, Italy

(C) 2020 Elsevier B.V. All rights reserved.

\title{
Uncertainty Analysis and Model Reduction Based Global Optimisation of Distributed Large-scale Systems
}

\author{
Min Tao, Constantinos Theodoropoulos \\ Department of Chemical Engineering and Analytical Science, University of \\ Manchester, Manchester M13 9PL, UK \\ k.theodoropoulos@manchester.ac.uk
}

\begin{abstract}
Uncertainty arises in many large-scale distributed industrial systems, needing efficient computational tools. Uncertainty propagation techniques have been developed and applied including power series expansions (PSE) and polynomial chaos expansions (PCE). However, such fast low-order approximate models generate errors and, in general, require prior knowledge about uncertainty distribution. In this work, the recursive projection method (RPM) was adopted to accelerate the computation of steady state solutions of complex large-scale dynamic systems. These accelerated models including uncertainty were subsequently utilised in an efficient Bayesian global optimisation framework. The performance of the proposed robust optimisation framework was demonstrated through an illustrative example: a tubular reactor where an exothermic reaction takes place.
\end{abstract}

Keywords: Uncertainty analysis, Distributed parameter systems, Recursive projection method, Bayesian global optimisation

\section{Introduction}

Complex distributed-parameter systems are common in industrial practice. Due to the inherent stochasticity and/or insufficient knowledge about processes, uncertainty arises (Eldred, 2009). In particular, parametric uncertainty in system models can greatly impact output performance. To robustly account for the impact of parametric uncertainties, uncertainty propagation techniques have been developed. Power series and polynomial chaos expansions methods have been used to address uncertainty through the application of low-order models, which worked efficiently for robust control (Nagy and Braatz, 2007). However, low-order approximate models may require prior knowledge about the distributions of parametric uncertainty (Kimaev and Richardez-Sandoval, 2018). For example, polynomial chaos expansion is implemented through "suitable" orthogonal polynomials corresponding to the uncertainty distribution (Xiu, 2010), which significantly affects computational accuracy. From the view of computational accuracy and generalised ability to handle uncertainty, directly using the original system model could be a better choice. To deal with intensive computational requirements of dynamic system models, the recursive projection method (Shroff and Keller, 1993) is used here. RPM has been multiply implemented as an effective model reduction technique for input/output systems for optimization and control purposes (Luna-Ortiz and Theodoropoulos, 2005; Bonis et al, 2013). RPM requires only a few utilisations of the (black-box) time integrator, to accelerate the computation of steady states through the calculation of low-order system Jacobians. Nevertheless, the 
computation of low-order derivatives including uncertainty is costly. Kriging surrogate model and Bayesian optimisation strategies are efficient for the expensive black-box systems (Jones et al, 1998). In this work, uncertainty analysis and robust optimisation framework have been constructed for large-scale input/output systems. Firstly, a RPMbased methodology was proposed to propagate parametric uncertainty in an accelerated way. Subsequently, the accelerated model with uncertainty was used by kriging models and a Bayesian global optimisation procedure to produce a robust optimal solution.

\section{Uncertainty Analysis}

\subsection{Large-scale distributed system with parametric uncertainty}

This work deals with the optimization of large-scale spatially distributed processes, described by a set of nonlinear dissipative PDEs with uncertainty:

$$
\frac{\partial X}{\partial t}=D\left\{\frac{\partial X}{\partial y}, \frac{\partial^{2} X}{\partial y^{2}}, \ldots, \frac{\partial^{n} X}{\partial y^{n}}\right\}+E\left(X, P_{1}, P_{2}\right)
$$

Where $D$ is the dissipative spatial differential operator, $E\left(X, P_{1}, P_{2}\right)$ is the nonlinear function, $X$ is the set of state variables, $P_{1} \in R^{n 1}$ are the design variables and $P_{2} \in R^{n 2}$ are the uncertain parameters.

\subsection{Recursive projection method (RPM)}

The dynamic systems in Eq. (1) are discretised and solved by a fixed-point procedure:

$U^{n+1}=F\left(U^{n}, P_{1}, P_{2} ; \tau\right)$

Where $U \in R^{N}$ is the discretised unknown variable, $n$ is the number of iteration steps, $F: R^{N} \times R^{n 1} \times R^{n 2} \rightarrow R^{N}, \tau$ is the reporting horizon of the black-box simulator.

The steady state solutions of the above fixed-point procedure are also the steady state solutions of the dynamic system:

$X=f\left(X, P_{1}, P_{2}\right)$

The recursive fixed-point procedure is time-consuming. To accelerate the computation, RPM is employed to decompose the solution space $R^{N}$ into two subspaces: the lowdimensional $P$ and its orthogonal complement $Q$ :

$R^{N}=P \oplus Q$

The orthonormal basis $Z$ of the slow subspace $P$ can be efficiently computed by matrix-free algorithms, such as the Arnoldi method. Then:

$I=Z^{T} Z, P=Z Z^{T}$

Where $I$ is the identity matrix, $Z \in R^{N \times m}$ with $m$ being the dimension of $P$.

The low dimensional Jacobians of subspace $P$ can then be approximated by directional perturbations:

$F_{U} Z=\frac{1}{2 \varepsilon}(F(U+\varepsilon Z)-F(U-\varepsilon Z))$ 
Where $\varepsilon$ is a small perturbation on the direction of $Z$.

Then the low-order Jacobian is given by:

$H=Z^{T} F_{U} Z$

Assuming the numerically stable time integration, the main RPM steps included the solution space decomposition (Eq. (8)), repeating Newton-Picard iterations (Eq. (9-10)) on the two subspaces and Picard iteration on the final sum to check the convergence $\left\|U\left(P_{1}, P_{2}\right)-F\left(U, P_{1}, P_{2}\right)\right\|$.

$p^{(0)}=P U^{(0)}\left(P_{1}, P_{2}\right), \quad q^{(0)}=Q U^{(0)}\left(P_{1}, P_{2}\right)$

$p^{(u+1)}=p^{(u)}+\left(I-Z H Z^{T}\right)^{-1} \times\left(P F\left(U^{(u)}, P_{1}, P_{2}\right)-p^{(u)}\right)$

$q^{(u+1)}=Q F\left(U^{(u)}, P_{1}, P_{2}\right)$

$U^{(u+1)}\left(P_{1}, P_{2}\right)=p^{(u+1)}+q^{(u+1)}$

Where $u$ is the number of Newton-Picard iterations.

Here, the Monte Carlo (MC) sampling method was used to approximate the parametric uncertainty. Therefore, RPM coupled with MC sampling method together accelerate the computation of the parametric uncertainty at steady state. The computationallyaccelerated model was subsequently applied in a Bayesian optimisation framework.

\section{Bayesian optimisation}

With the acceleration technique RPM, optimising the system model with uncertainty is still costly. In this work, Bayesian optimisation strategy was adopted through kriging model and expected improvement function, originating from efficient global optimisation (Jones et al, 1998).

3.1. Kriging model

Kriging model, a popular surrogate models, assumes that the random output variable $f(y)$ at any location point $y$ with mean value $\mu$ and variance $\sigma^{2}$, and prior joint Gaussian distribution for the finite random variables:

$f(Y) \sim N(\mu(Y), K(Y, Y))$

Where $f(Y)=\left(f\left(y_{1}\right), f\left(y_{2}\right) \ldots f\left(y_{N}\right)\right), \quad \mu(Y)=\left(\mu\left(y_{1}\right), \mu\left(y_{2}\right) \ldots \mu\left(y_{N}\right)\right)$ and $K_{i j}=$ $k\left(y_{i}, y_{j}\right), k\left(y_{i}, y_{j}\right)$ is the kernel function.

Here, Gaussian kernels are used:

$k\left(y_{i}, y_{j}\right)=\sigma_{f}^{2} \exp \left(-\frac{1}{2 l^{2}}\left(y_{i}-y_{j}\right)^{T}\left(y_{i}-y_{j}\right)\right)$

Where kernel parameters $\sigma_{f}^{2}$ and $l$ are computed by a few initial observed samples.

The observed variables $f(Y)$ and unknown variables $f_{*}\left(Y_{*}\right)$ given new points $Y_{*}$ satisfy a joint Gaussian distribution:

$\left(\begin{array}{c}f(Y) \\ f_{*}\left(Y_{*}\right)\end{array}\right) \sim N\left(\begin{array}{c}\mu(Y) \\ \mu\left(Y_{*}\right)\end{array}\right),\left(\begin{array}{cc}K & k\left(Y, Y_{*}\right) \\ k\left(Y_{*}, Y\right) & k\left(Y_{*}, Y_{*}\right)\end{array}\right)$

The posterior predictive $f_{*}\left(Y_{*}\right)$ is still a Gaussian process:

$f_{*} \mid\left(f(Y), Y, Y_{*}\right) \sim N\left(\mu_{*}, \sigma_{*}^{2}\right)$ 
Where $\mu_{*}=\mu\left(Y_{*}\right)+k\left(Y_{*}, Y\right) K^{-1}(f(Y)-m(Y)), \sigma_{*}^{2}=k\left(Y_{*}, Y_{*}\right)-k\left(Y_{*}, Y\right) K^{-1} k\left(Y, Y_{*}\right)$

\subsection{Expected improvement (EI) function}

In Bayesian global optimisation, acquisition functions decide the next sampling point. Here, EI function under kriging model is used to balance exploitation with exploration:

$E I(y)=\left\{\begin{array}{cc}\left(\mu(y)-f\left(y^{+}\right)-\varphi\right) \Phi(g)+\sigma(y) \phi(g) & \sigma(y)>0 \\ 0 & \sigma(y)=0\end{array}\right.$

Where $g=\left\{\begin{array}{cc}\frac{\mu(y)-f\left(y^{+}\right)-\varphi}{\sigma(y)} & \sigma(y)>0 \\ 0 & \sigma(y)=0\end{array}\right.$

Here $\mu(y)$ and $\sigma(y)$ are the mean and the standard deviation of the kriging model, respectively. $\Phi$ and $\phi$ are the CDF and PDF of the standard normal distribution, respectively. $y^{+}$is the location of current best sample and $\varphi$ is a parameter that determines the amount of exploration.

\section{Application}

The effectiveness of the proposed optimisation framework is illustrated using an exothermic tubular reactor (Tao et al, 2019). The mathematical formulation is as follows:

$\max _{T w i} a \mu\left(C_{e x i t}\right)-(1-a) \sigma\left(C_{\text {exit }}\right)$

s.t. $\frac{\partial C}{\partial t}=\frac{1}{P e_{1}} \frac{\partial^{2} C}{\partial y^{2}}-\frac{\partial C}{\partial y}+D a(1-C) \exp (T /(1+T / \gamma))$

$\frac{\partial T}{\partial t}=\frac{1}{P e_{2}} \frac{\partial^{2} T}{\partial y^{2}}-\frac{\partial T}{\partial y}-\beta T+B D a(1-C) \exp (T /(1+T / \gamma))+\beta T_{w}$

$T_{w}(y)=\sum_{i=1}^{3}\left(H\left(y-y_{i-1}\right)-H(y)-y_{i}\right) T_{w i}$

$D a \sim N(0.1,0.01)$

Here $C$ and $T$ are the dimensionless concentration and temperature respectively, $C_{\text {exit }}$ the dimensionless output concentration, $\mu\left(C_{\text {exit }}\right)$ is the mean value of the uncertain output $C_{\text {exit }} . \sigma\left(C_{\text {exit }}\right)$ is the standard variance value of $C_{\text {exit }} . T_{w}$ is the adiabatic wall temperature at the three cooling zones. $H$ is the Heaviside step function. The system parameters are $P e_{1}=5, P e_{2}=5, \beta=1.5, \gamma=10, B=12 . D a$ is the Damkohler number.

In this optimisation problem, the objective is to maximize the average value and minimize the variance of the output dimensionless product concentration, with uncertainty parameter $D a$ and design variables $T_{w} . a$ is the weight between the two terms, here set to be 0.6 . The system model is given by a dynamic black-box simulator with inputs the design variables and the uncertain parameter and outputs the dimensionless concentration and temperature. The RPM algorithm was implemented in MATLAB R2019a and Bayesian optimisation in Python 3.7.3/PyCharm 2018.3.5 on a Desktop ((Intel Core(TM) i7-8700 CPU 3.2 GHz, 16 GB memory, 64-bit operating system, Windows 10). 


\subsection{Uncertainty analysis}

To investigate the effect of parametric uncertainty on the output concentration, the uncertainty propagation was constructed. Firstly, the number of MC samples was decided to be 3000 by an independent numerical experiment. Then $3000 \mathrm{MC}$ samples were generated to approximate the parameter distribution. To compare results of different uncertainty propagation methods, the same $3000 \mathrm{MC}$ samples were utilized with the original dynamic solver, RPM using the dynamic solver, second-order PSE and second-order PCE. The report horizon was 0.01 seconds the dimension of the dominant subspace was fixed to 10 for the RPM case. The perturbation, $\varepsilon$, was chosen to be 0.01 for PSE after numerical experiments with different perturbation sizes. 9 points of the original system were used for PCE. Table 1 shows that direct propagation uncertainty through the original dynamic solver is the most time-consuming. RPM significantly accelerated the computations with more than $87 \%$ saving and almost the same accuracy (also displayed in Figure 1). Although PSE took the least computational time, choosing the perturbation size increased the actual total cost to be 14.48 minutes. Compared with PSE, PCE produced more accurate mean value, low and upper bounds.Table 1 also shows the large variance of PSE and PCE because of missing a small spatial distribution as displayed in Figure 1.

Table 1 . Uncertainty analysis ( $5 \%$ confidence level)

\begin{tabular}{|c|c|c|c|c|c|}
\hline Models & $\begin{array}{l}\text { Low bound } \\
C_{\text {exit }}\end{array}$ & $\begin{array}{l}\text { Up bound } \\
C_{\text {exit }}\end{array}$ & $\begin{array}{l}\text { Mean } \\
C_{\text {exit }}\end{array}$ & $\begin{array}{l}\text { Standard } \\
\text { variance } \\
C_{\text {exit }}\end{array}$ & $\begin{array}{l}\text { Computational } \\
\text { time (minutes) }\end{array}$ \\
\hline $\begin{array}{l}\text { Dynamic } \\
\text { solver }\end{array}$ & 0.1204 & 0.3136 & 0.1963 & 0.0999 & 319.24 \\
\hline \multirow{2}{*}{$\begin{array}{l}\text { RPM plus } \\
\text { Dynamic } \\
\text { solver }\end{array}$} & 0.1204 & 0.3136 & 0.1963 & 0.0999 & \multirow[t]{2}{*}{40.49} \\
\hline & Error : $0 \%$ & Error : $0 \%$ & $\begin{array}{l}\text { Error : } \\
0 \%\end{array}$ & Error : $0 \%$ & \\
\hline \multirow[t]{2}{*}{ PSE } & 0.1266 & 0.2856 & 0.1869 & 0.0418 & 4.29 \\
\hline & Error: $5.15 \%$ & $\begin{array}{l}\text { Error : - } \\
8.93 \%\end{array}$ & $\begin{array}{l}\text { Error : - } \\
4.79 \%\end{array}$ & Error : $\mathbf{- 5 8 . 1 6 \%}$ & \\
\hline \multirow[t]{2}{*}{ PCE } & 0.1215 & 0.3018 & 0.1876 & 0.0478 & 8.71 \\
\hline & Error: $\mathbf{- 0 . 9 1 \%}$ & $\begin{array}{l}\text { Error: } \\
-3.76 \%\end{array}$ & $\begin{array}{l}\text { Error: - } \\
\mathbf{4 . 4 3} \%\end{array}$ & Error: -52.15 \% & \\
\hline
\end{tabular}

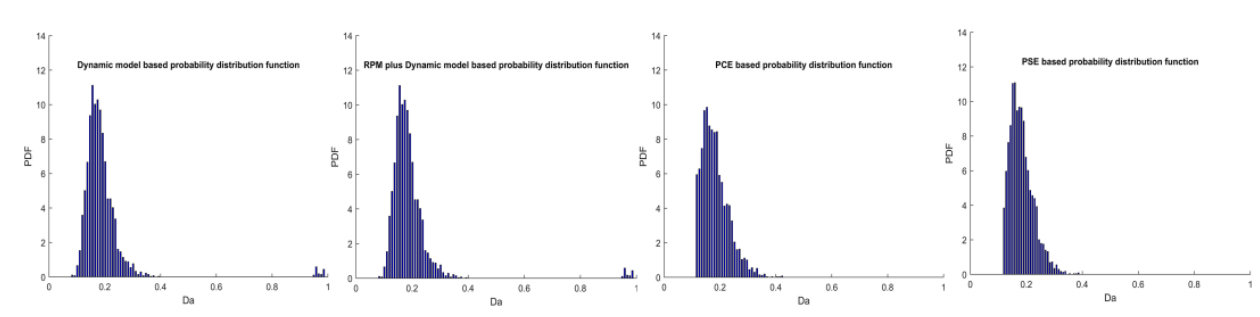

Figure 1. Probability density functions of the steady state output concentration

\subsection{Bayesian optimisation}

Using RPM with the black-box dynamic solver, Bayesian optimisation was constructed for the problem (Eq. (17)). Latin hypercube sampling method was utilised, taking 9.75 
hours for the 8 initial samples. The optimisation process, as showed in Figure 2, converged to the optimum solution within 33 iterations and 30.91 hours.

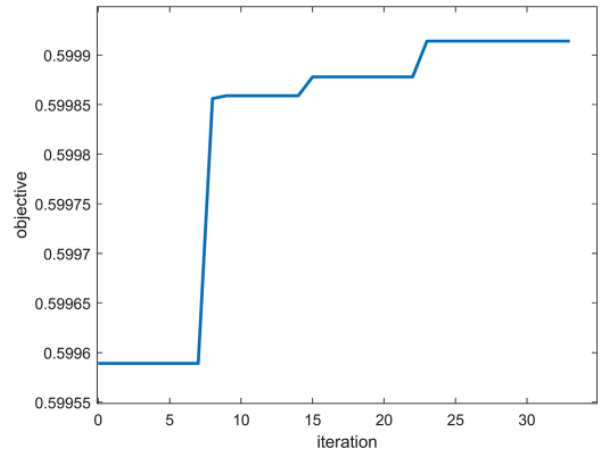

Figure 2. Bayesian optimisation of cooling process with parametric uncertainty

\section{Conclusions}

This paper presents a robust steady-state optimisation framework for large-scale dynamic systems with parametric uncertainty. RPM is employed to accelerate the dynamic systems to be steady state, resulting in significant cost reduction of uncertainty propagation. Furthermore, Bayesian optimisation with kriging model is utilised to find the optimum of the costly system within an acceptable number of iteration steps. The tubular reactor case study verifies the efficiency of our framework. In the future, the optimisation framework will be applied to scenarios of multivariate uncertainty and large-scale distributed stochastic systems.

\section{References}

M. Eldred, 2009, Recent advances in non-intrusive polynomial chaos and stochastic collocation methods for uncertainty analysis and design, In 50th AIAA/ASME/ASCE/AHS/ASC Structures, Structural Dynamics, and Materials Conference 17th AIAA/ASME/AHS Adaptive Structures Conference 11th AIAA No (p. 2274).

Z.K. Nagy, R.D. Braatz, 2007, Distributional uncertainty analysis using power series andlynomial chaos expansions, Journal of Process Control, 17(3), pp.229-240.

G. Kimaev, L.A. Ricardez-Sandoval, 2018, Multilevel Monte Carlo applied to chemical engineering systems subject to uncertainty. AIChE Journal, 64(5), pp.1651-1661.

G.M. Shroff, and H.B. Keller, 1993, Stabilization of unstable procedures: the recursive projection method, SIAM Journal on numerical analysis, 30(4), pp.1099-1120.

E. Luna-Ortiz, C. Theodoropoulos, 2005, An input/output model reduction-based optimization scheme for large-scale systems, Multiscale Modeling \& Simulation, 4(2) pp. 691-708.

I. Bonis, W. Xie, C. Theodoropoulos, 2013, Multiple model predictive control of dissipative PDE systems, IEEE Transactions on Control Systems Technology, 22(3) pp. 1206-1214.

D.R. Jones , M. Schonlau, W.J. Welch, 1998, Efficient global optimization of expensive blackbox functions, Journal of Global optimization, 13(4), pp.455-492.

M. Tao, J. Li, C. Theodoropoulos, 2019, Reduced model-based global optimisation of large-scale steady state nonlinear systems, In Computer Aided Chemical Engineering (Vol. 46, pp. 10391044). Elsevier. 